\title{
Bitcoin Transaction Networks: An Overview of Recent Results
}

\author{
Nicoló Vallarano ${ }^{1}$, Claudio J. Tessone ${ }^{2,3 *}$ and Tiziano Squartini ${ }^{1}$ \\ ${ }^{1}$ IMT School of Advanced Studies, NETWORKS Unit, Lucca, Italy, ${ }^{2}$ UZH Blockchain Center, University of Zürich, Zurich, \\ Switzerland, ${ }^{3}$ URPP Social Networks, University of Zürich, Zurich, Switzerland
}

Cryptocurrencies are distributed systems that allow exchanges of native (and non-) tokens between participants. The availability of the complete historical bookkeeping opens up an unprecedented possibility: that of understanding the evolution of a cryptocurrency's network structure while gaining useful insights into the relationships between users' behavior and cryptocurrency pricing in exchange markets. In this article we review some recent results concerning the structural properties of the Bitcoin Transaction Networks, a generic name referring to a set of three different constructs: the Bitcoin Address Network, the Bitcoin User Network, and the Bitcoin Lightning Network. The picture that emerges is of a system growing over time, which becomes increasingly sparse and whose mesoscopic structural organization is characterized by the presence of an increasingly significant core-periphery structure. Such a peculiar

OPEN ACCESS

Edited by:

Marco Alberto Javarone, University College London,

United Kingdom

Reviewed by:

Chengyi Xia,

Tianjin University of Technology, China Matúš Medo,

University of Electronic Science and Technology of China, China

*Correspondence:

Claudio J. Tessone

claudio.tessone@business.uzh.ch

Specialty section:

This article was submitted to Social Physics,

a section of the journal

Frontiers in Physics

Received: 24 April 2020

Accepted: 25 June 2020

Published: 03 December 2020

Citation:

Vallarano $\mathrm{N}$, Tessone $\mathrm{CJ}$ and

Squartini T (2020) Bitcoin Transaction Networks: An Overview of Recent

Results. Front. Phys. 8:286.

doi: 10.3389/fphy.2020.00286 topology is accompanied by a highly uneven distribution of bitcoins, a result suggesting that Bitcoin is becoming an increasingly centralized system at different levels.

Keywords: blockchain, bitcoin, complex networks, centralization, inequality, null models

\section{INTRODUCTION}

A cryptocurrency is an online payment system for which the storage and verification of transactions-and therefore the safeguarding of the system's consistency itself-are decentralized, i.e., do not require the presence of a trusted third party. This result can be achieved by securing financial transactions through a clever combination of cryptographic technologies [1].

Bitcoin, the first and most popular cryptocurrency, was introduced in 2008 by Satoshi Nakamoto [2]. It consists of a decentralized peer-to-peer network to which users connect to exchange property in the account units of the system, i.e., to perform transactions of bitcoins. Each transaction becomes part of a publicly available ledger, the blockchain, after having been validated by so-called miners, i.e., users who verify the validity of issued transactions according to the consensus rules that are part of the Bitcoin protocol $[3,4]$. A new block, containing transactions known to the miner since the last block, is "mined" every 10 min on average, thereby adding new transactions to the blockchain; thus these transactions are "confirmed," in turn enabling users to spend the bitcoins they received through them ${ }^{1}$. The cryptography protocols that Bitcoin rests upon aim to prevent the so-called double-spending problem, i.e., the possibility of the same digital token being spent more than once in the absence of a central party that guarantees the validity of the transactions $[1,2]$; remarkably, the transaction-verification mechanism Bitcoin relies on allows its entire transaction history to be openly accessible, a feature that, in turn, allows researchers to analyze Bitcoin transactions in different network representations.

\footnotetext{
${ }^{1}$ In practice, the so-called " 6 confirmations" rule is followed: once a transaction is included in a block that is followed by at least six additional blocks [5], the transaction can be safely considered confirmed.
} 
The gain in Bitcoin's popularity has given rise to new problems for its community, including (i) the lack of scalability of the transaction-verification method, i.e., the relatively low maximum number of transactions that can be verified per second, especially when compared with mainstream competitors, such as centralized payment networks, (ii) the increased concentration of mining power in mining pools, which implies that the verification mechanism in the network is becoming less and less distributed, and (iii) the tendency of users to hoard. In order to overcome these problems, which threaten the overall functioning of Bitcoin as a medium of exchange, new instruments have been adopted. Proposed in 2015 [6], the Bitcoin Lightning Network (BLN) is a "Layer 2" protocol that can operate on top of blockchain-based (Bitcoin-like) cryptocurrencies by creating bilateral channels for off-chain payments which are then settled concurrently on the blockchain, once the channels are closed. As both the transaction fees and the blockchain confirmation are no longer required, the network is spared from avoidable burdens; moreover, the key features of Bitcoin, i.e., its decentralized architecture, its political organization, and its wealth distribution, are no longer sacrificed, while the circulation of the native assets is enhanced.

Bitcoin is almost 10 years old; however, while a large amount of literature concerning either the purely financial or the purely engineering aspects of it exists (e.g., prediction of the exchange rate between Bitcoin and the US dollar [7], statistical properties of the exchange rate [8], statistical properties of Bitcoin daily log-returns [9], comparison of Bitcoin volatility with that of the exchange rates of major global currencies $[10,11]$, identification of factors influencing the Bitcoin price [12], predictability of the Bitcoin price via machine-learning techniques [13], the interplay between social interactions and movements of the Bitcoin price $[14,15]$, and the problem of de-anonymization of Bitcoin users [16-20]), only recently have researchers started to investigate the structural properties of Bitcoin. In Kondor et al. [21], the authors consider the network of transactions between addresses at the weekly time scale, showing the emergence of power-law distributions and that the number of incoming transactions reflects the wealth of nodes; in Javarone and Wright [22], the network of transactions between users is studied at the macroscale, in order to check for its small-worldness; in Parino et al. [23], the authors investigate the network of international Bitcoin flows, identifying socio-economic factors that drive Bitcoin adoption across countries. In general, however, the works analyzing Bitcoin from a network perspective provide a quite limited view of its evolution, focusing either on a single representation of the network or on a relatively short period of time; even those studies that address the problem from a wider perspective $[24,25]$ are often limited to a purely descriptive analysis and do not compare empirical observations with the outcomes of proper models.

In this article we summarize the results of three papers [26-28], providing a comprehensive overview of the empirical traits that characterize Bitcoin evolution and framing them within models rooted in statistical physics. In Bovet et al. [26], the authors analyzed the local properties of two Bitcoin representations, the Bitcoin Address Network (BAN) and the Bitcoin User Network (BUN), and looked for the presence of correlations between (exogenous) price movements and (endogenous) changes in the topological structure of the networks. In Bovet et al. [27], the mesoscale structure of the BUN is examined; particular attention is paid to identifying the best network model able to describe the structure; in addition, the same exercise as above is carried out, i.e., comparison of the evolution of purely structural properties and the appearance of price bubbles in a cyclical fashion. Lastly, in Lin et al. [28], the evolution of the BLN's topology is investigated, revealing that the BLN is becoming an increasingly centralized system and that the "capital" is becoming increasingly unevenly distributed.

\section{DATA}

As previously mentioned, Bitcoin relies on a decentralized public ledger, the blockchain, that records all transactions between Bitcoin users. A transaction is a set of input and output addresses; the output addresses that are "unspent," i.e., not yet recorded on the ledger as input addresses, can be claimed-and therefore spent-only by the owner of the corresponding cryptographic key. This is the reason one speaks of pseudonimity: an observer of the blockchain can see all unspent addresses but cannot link them to the actual owners.

\subsection{The Bitcoin Address Network (BAN)}

The BAN is the simplest network that can be constructed from the blockchain records. From a technical point of view, it is a directed weighted graph whose nodes represent addresses; the directions and weights of the links between nodes are provided by the input-output relationships defining the transactions recorded on the blockchain. The BAN was considered over a period of 9 years, from 9th January 2009 to 18th December 2017, at the end of which the data set consisted of 304,111,529 addresses, between which a total number of $283,028,575$ transactions were performed. In terms of traded volume, the transactions between addresses amounted to 4,432,597,496 bitcoins.

\subsection{The Bitcoin User Network (BUN)}

Since the same owner may control several addresses [18], one can define a network of "users" whose nodes are clusters of addresses. These clusters are derived by implementing different heuristics provided by the state-of-the-art literature [16, 17, 29, 30]. The "user networks" thus obtained should not be regarded as perfect representations of the actual networks of users, but rather as attempts to group addresses while minimizing the presence of false positives. Two heuristics have been employed here: the multi-input one (based on the assumption that addresses appearing as input to the same transaction are controlled by the same user) and the change address one (based on the assumption that a new address appearing as output of a transaction and with the smallest amount of transferred money must belong to the input user). The BUN was considered over the same period as the BAN (i.e., 9 years from 9th January 2009 to 18 th December 2017), at the end of which the data set consisted of $16,749,939$ users, between which a total number of 224,620,265 transactions took place. In terms of traded volume, the transactions between users amounted to 3,114,359,679 bitcoins. 


\subsection{The Bitcoin Lightning Network (BLN)}

The BLN is constructed similarly to the way the BAN is defined. It is a directed weighted graph whose nodes are addresses exchanging bitcoins on "Layer 2." Three different representations of the BLN have been studied so far: the daily, the weekly, and the daily-block representations. While a daily/weekly snapshot includes all channels that were found to be active during that day/week, a daily-block snapshot consists of all channels that were found to be active at the time the first block of the day was released (hence, the transactions considered for the dailyblock representation are a subset of those constituting the daily representation). The BLN was considered over a period of 18 months, from 14th January 2018 to 13th July 2019, at the end of which the network consisted of 8,216 users, 122,517 active channels, and 2732.5 transacted bitcoins.

\subsection{Notation}

Although information about the magnitude of transactions is available, the BAN and the BUN were analyzed as binary directed networks; as such, they are completely specified by their binary asymmetric adjacency matrices, $\mathbf{A}_{\mathrm{BAN}}^{(t)}$ and $\mathbf{A}_{\mathrm{BUN}}^{(t)}$, at time $t$. The generic entry $a_{i j}^{(t)}$ is equal to 1 if at least one transaction between address (user) $i$ and address (user) $j$ takes place, i.e., if bitcoins are transferred from address (user) $i$ to address (user) $j$, during the time snapshot $t$ and is equal to 0 otherwise. The BLN, on the other hand, is a weighted undirected network, represented by a symmetric matrix $\mathbf{W}_{\mathrm{BLN}}^{(t)}$ whose generic entry $w_{i j}^{(t)}=w_{j i}^{(t)}$ indicates the total amount of money exchanged between $i$ and $j$, across all channels, at time $t$; here, we will focus mainly on its binary projection $\mathbf{B}_{\mathrm{BLN}}^{(t)}$, whose generic entry is $b_{i j}^{(t)}=b_{j i}^{(t)}=1$ if $w_{i j}^{(t)}=w_{j i}^{(t)}>0$ and $b_{i j}^{(t)}=b_{j i}^{(t)}=0$ otherwise.

\section{RESULTS}

\subsection{The Bitcoin Address Network and the Bitcoin User Network}

Let us start by reviewing results on the BAN and the BUN at the weekly time scale. Similar results have been obtained for the BAN and the BUN at the daily time scale [26].

\subsubsection{Basic Statistics}

We begin by commenting on the evolution of some basic statistics characterizing the BAN and BUN that, as noted elsewhere [19], have started to evolve in a more stationary fashion since mid-2011. As Figure 1 shows, both the number of nodes $N$ and the number of links $L=\sum_{i} \sum_{j(\neq i)} a_{i j}$ increase steadily over time, irrespective of the representation considered; the link density $d=\frac{L}{N(N-1)}$, however, decreases, meaning that the system is becoming sparser. The dependence of $d$ on $N$ can be better specified, from a mathematical point of view, upon observing that the average degree $\overline{k^{\text {in }}}=\overline{k^{\text {out }}}=\frac{\sum_{i} \sum_{j(\neq i)} a_{i j}}{L}=\frac{L}{N}$ is either constant (for the BUN) or limited (for the BAN) over time [26]; hence, it follows that $L \propto N$ and $d \sim N^{-1}$.

\subsubsection{Degree Distributions}

Generally speaking, both out-degrees and in-degrees are characterized by heavy-tailed distributions, indicating that a large number of low-connectivity nodes coexist with a few hubs whose degree is several orders of magnitude greater. A visual inspection of the functional form of the degree distributions suggests that the out-degrees distribution follows a power law [26, 31]. To test this hypothesis Bovet et al. [26] employed an algorithm based on a double Kolmogorov-Smirnov statistical test [32]; they found that the hypothesis above cannot be rejected, at a 0.05 confidence level, for almost half of the considered snapshots.

Of particular interest is the evolution of the out-degrees standard deviation, especially in regard to its informativeness about exogenous events. As an example, consider the failure in February 2014 of Mt. Gox, a quasi-monopolist exchange market at the time. This event deeply affected the overall Bitcoin structure: the percentage of snapshots for which the null hypothesis (that the out-degrees distribution follows a power law) can be rejected was $\simeq 50 \%$ before February 2014 and dropped to $\simeq 25 \%$ afterwards.

Bovet et al. [26] also argued that the presence of heavy-tailed distributions may be explained by a mechanism similar to that of preferential attachment: new, or occasional, users "preferentially" connect to already well-connected nodes (exchange markets, utility providers, etc.), thus leading to the formation of superhubs. Elsewhere it has been argued that the related mechanism known as "fittest-gets-richer" or "good-gets-richer" [33] may be also at work, with the computational resources of a node playing the role of its fitness [22].

\subsubsection{Bitcoin Structure vs. Bitcoin Price}

The result concerning the evolution of the out-degrees distribution suggests that the Bitcoin network structure indeed carries signatures of exogenous events. As in this case, the nonstructural quantity par excellence is represented by the price of the currency, it may be of interest to look for the presence of correlations between the evolution of the price and the evolution of purely topological quantities. Justification for such an analysis rests upon the simple consideration that the price of a cryptocurrency is ultimately related to the behavior of users whose "network" activity translates into that of establishing connections with other nodes, whence our expectation of finding some traces of the aforementioned correlations.

The simplest analysis is based on drawing scatterplots of the network size and network link density vs. the Bitcoin price (in USD). As Figure 2 shows, a clear trend appears, indicating that the price and the network size $N$ (respectively, the link density d) are overall positively (respectively, negatively) correlated throughout the entire Bitcoin history. Notice, however, the trend inversion that occurs immediately after the Mt. Gox failure; it is a consequence of the prolonged price decrease observed in 20142016, during which the network size increased by almost one order of magnitude.

To further confirm the presence of a double regime, Bovet et al. [26] inspected the correlation between the moments of the out-degrees distribution and the Bitcoin price over time. To this end, the so-called "ratio of price to its moving average" (RPMA) 


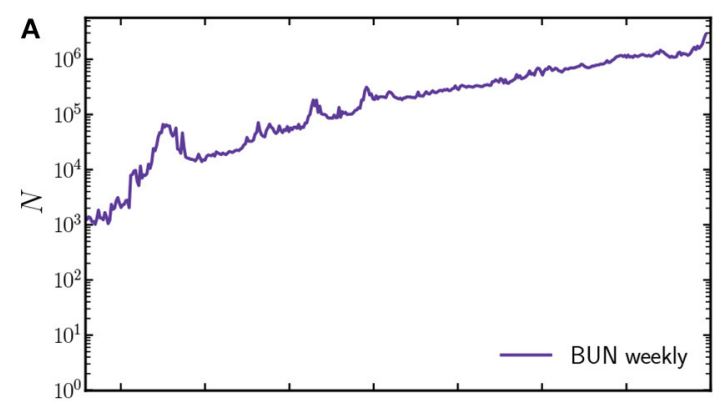

C

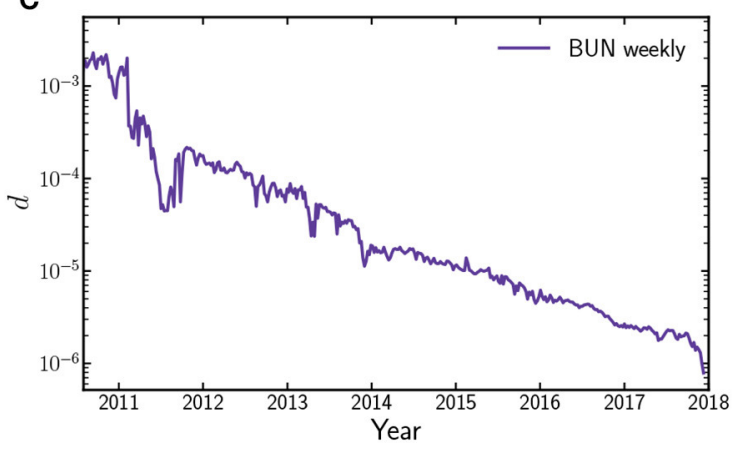

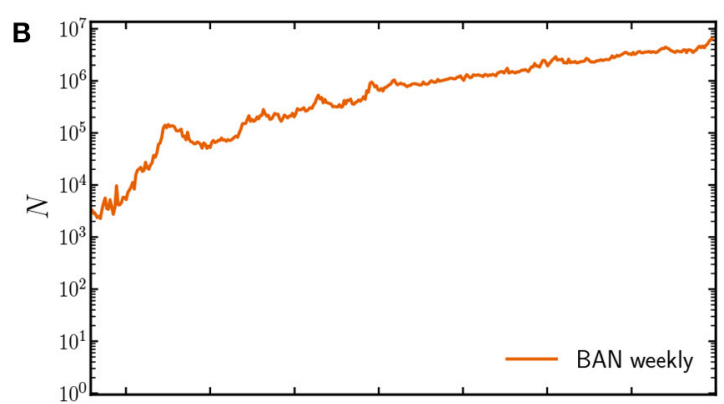

D

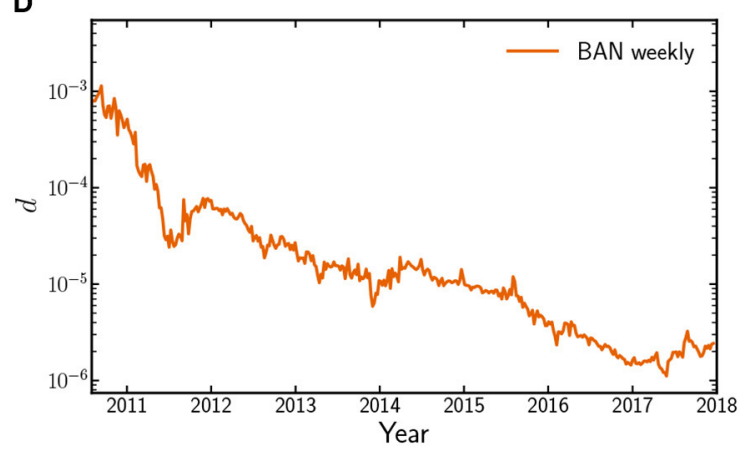

FIGURE 1 | Evolution of basic statistics, i.e., the number of nodes (A,B) and the link density (C,D) for two Bitcoin network representations, i.e., the BAN (A,C) and the BUN (B,D) at the weekly time scale, from July 2010 to 18th December 2017 (i.e., for networks with at least 200 nodes). Although the network size increases, it becomes sparser (irrespectively from the considered representation). Similar results are observed for the BAN and the BUN at the daily time scale. See also Bovet et al. [26].

indicator was defined:

$$
\mathrm{RPMA}_{t}=100 \log _{10}\left(\frac{P_{t}}{\frac{1}{\tau} \sum_{s=t-1-\tau}^{t-1} P_{s}}\right)
$$

where $\tau$ represents a tunable temporal parameter. As shown in Bovet et al. [26], the standard deviation and the kurtosis diverge as the network size becomes larger than the value corresponding to the Mt. Gox failure, thus confirming the "two regimes" hypothesis. Moreover, as Figure 2 shows, larger values of the moments (observed after the Mt. Gox failure) correspond to price drops, while temporal snapshots corresponding to smaller values of the same quantities seem to be characterized by price increases.

A multivariate Granger test [34] was also carried out to unveil possible lagged correlations hidden in the data (see Figure 5 in Bovet et al. [26]). For this purpose, the data were split into two sub-samples, corresponding to the time periods 2010-2013 and 2014-2017, and the number of nodes $N$, the number of links $L$, and the higher moments of the empirical out- and in-degrees distributions were related to the log-returns of the Bitcoin price (in USD) within each sub-sample. To sum up, when the BUN is considered on the weekly time scale, a positive feedback loop occurs between $N$ and the price log-returns, whereas on the daily time scale a price increase predicts an increase in the number of nodes $N$ but not vice versa. The causality structure is consistent within the two sub-samples.

\subsubsection{Analysis of the BUN Mesoscale Structure}

We now review the results concerning the mesoscale structure of the BUN. A recently proposed method [35] based on the surprise score function was adopted by Bovet et al. [27] to assess the statistical significance of a peculiar mesoscale organization known as the core-periphery structure. According to the interpretation proposed in de Jeude et al. [35], revealing the core-periphery structure by minimizing the surprise means distinguishing the partition that is least likely to be explained by the null model known as the random graph model (RGM) relative to the null model known as the stochastic block model (SBM); see also Appendix A. As Figure 3 shows, a core-periphery structure is indeed present; more precisely, during 2014-2015 the core size amounts to $\simeq 30 \%$ of the total network size; but after 2016 it seems to shrink back to 2010-2013 levels. The presence of a core-periphery structure indicates that the BUN is characterized by subgraphs with very different link densities-evidence that cannot be accounted for by a model, such as the RGM, with just one global parameter.

A closer inspection of the BUN core-periphery structure reveals it to be even richer. In fact, the core portion of the BUN is the strongly connected component (SCC) of a bow-tie structure whose remaining portions (the IN and OUT components) make up the BUN periphery [27]. More specifically, while the SCC is the set of nodes that are mutually reachable (i.e., there exists a directed path from any node to any other node within the SCC), the IN and OUT components are defined, respectively, as the 

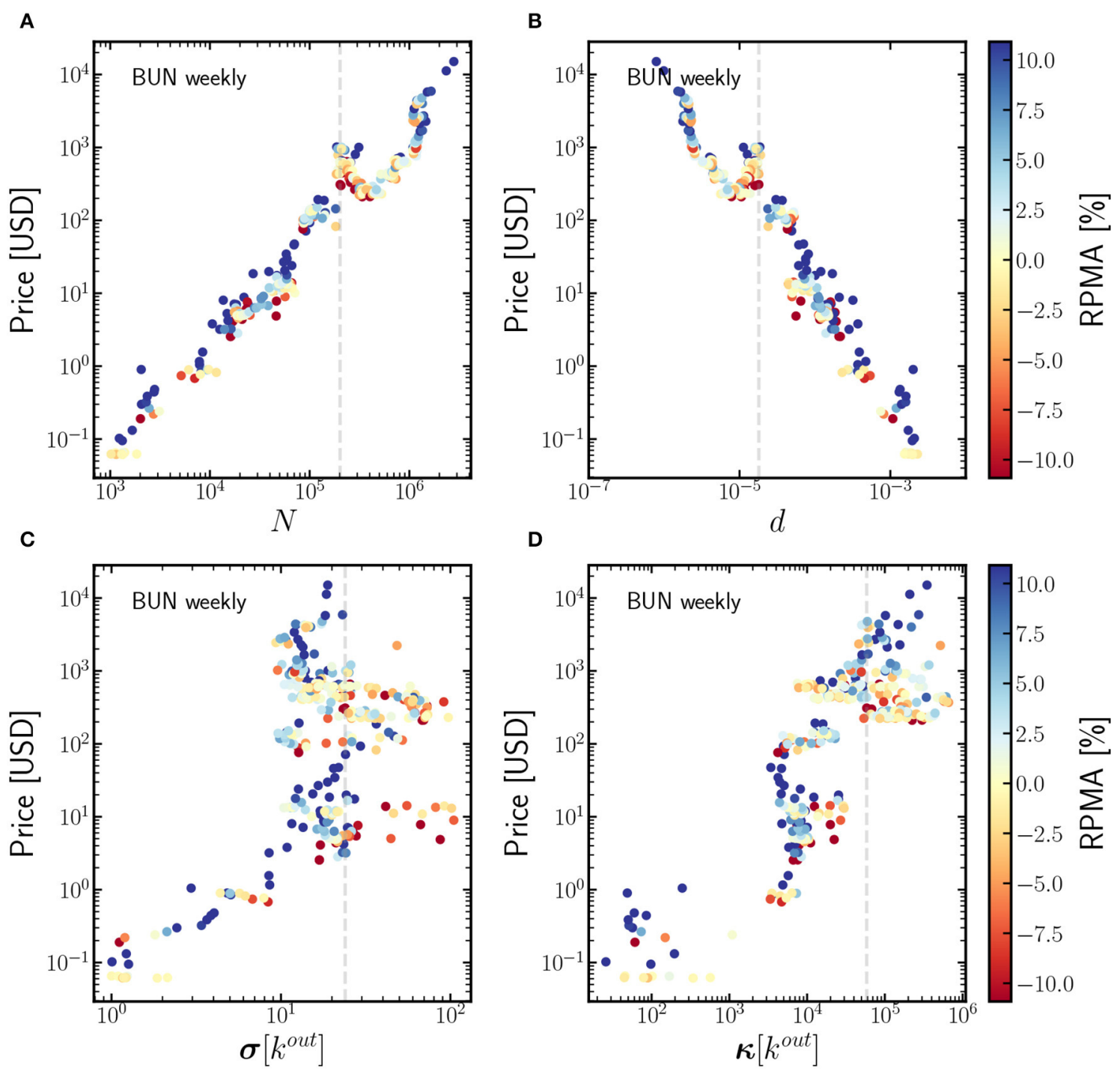

FIGURE 2 | Correlation between the Bitcoin price in USD, the basic statistics (number of nodes and link density -A,B) and the moments of the out-degrees distribution (C,D) for the BUN at the weekly time scale. Additionally, each dot representing an observation is colored according to the value of the Ratio between the current Price and its Moving Average (RPMA) indicator. The vertical, dashed line coincides with the bankruptcy of Mt. Gox. Purely structural quantities are correlated with exogenous quantities as the Bitcoin price; see, for example, the evolution of the out-degrees standard deviation whose larger values (observable after the Mt.

Gox failure) correspond to price drops. See also [26].

set of nodes from which the SCC can be reached and the set of nodes that can be reached from the SCC. Hence, the picture provided by the evolution of the core-periphery structure can be further refined as follows: since 2016 both the SCC and the OUT component have shrunk while the IN component has become the dominant portion of the network [27]. Other SCCs are visible but are negligibly small relative to the largest one, which seems to indicate that they are, in fact, single nodes pointing to (or pointed to by) hubs.

An additional analysis aimed at better quantifying the extent to which a generic, purely topological quantity $X$ and the Bitcoin price are related can be carried out by plotting the evolution of the temporal $z$-score

$$
z_{X}^{(t)}=\frac{X^{(t)}-\bar{X}}{s_{X}},
$$

where $\bar{X}=\sum_{t} \frac{X^{t}}{T}$ is the mean over a sample of values covering the period $T$ before time $t$ (in our case, the year before $t$ ) and $s_{X}=\sqrt{\overline{X^{2}}-\bar{X}^{2}}$ is the corresponding standard deviation. For example, the choice $X=\sigma\left[k^{\text {out }}\right]$ allows price drawdowns to be revealed and, in some cases, anticipated [26]; in the 3-years period 2010-2012, as well as after 2017, the price rises as $z_{\sigma\left[k^{\text {out }}\right]}^{(t)}$ increases, whereas drawdowns occur in periods during which 


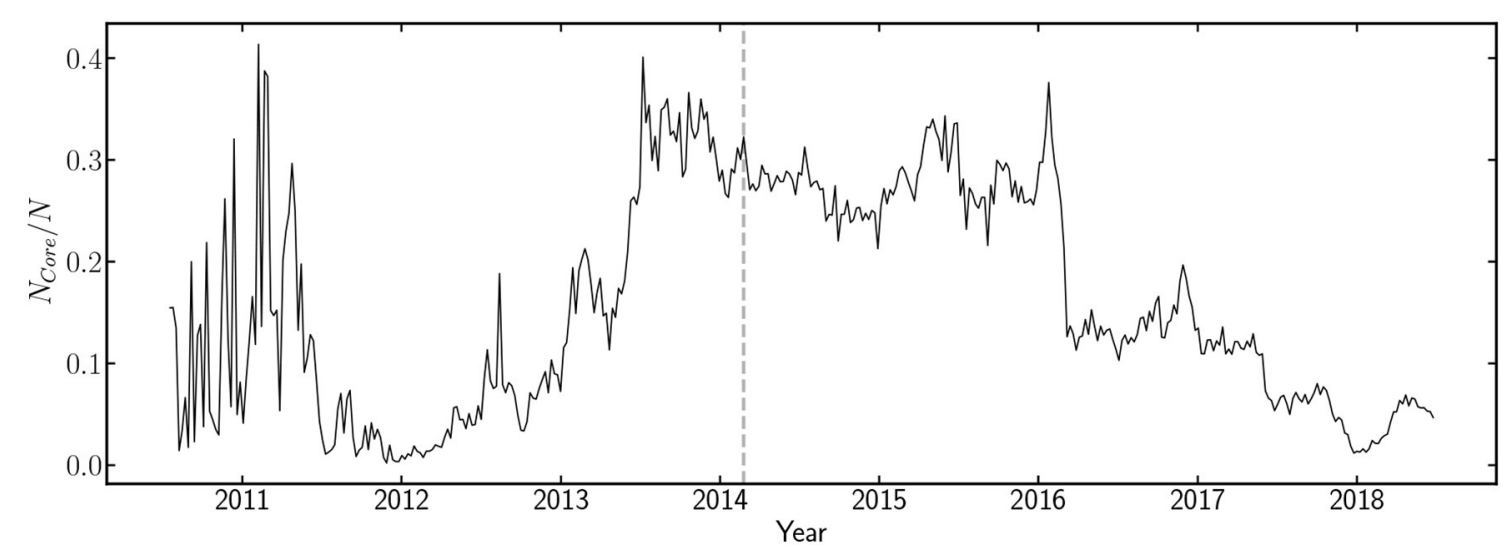

FIGURE 3 | Evolution of the percentage of nodes belonging to the core portion of the BUN on the weekly time scale. During $2012-2013$ the core portion of the BUN steadily rises until it reaches $\simeq 30 \%$ of the network; then, during 2014-2015, it remains fairly constant; during the last 2 years covered by our data set (2016-2018), the core portion of the BUN shrinks and the percentage of nodes belonging to it falls back to pre-2012 levels. The vertical dashed line coincides with the bankruptcy of Mt. Gox.

$z_{\sigma\left[k^{\text {out }}\right]}^{(t)}$ decreases. Other possible choices are $X=N_{\text {core }}$, the number of core nodes, and $X=r$, the network reciprocity, defined as $r=\frac{\sum_{i} \sum_{j(\neq i)} a_{i j} a_{j i}}{\sum_{i} \sum_{j(\neq i)} a_{i j}}$, i.e., the percentage of links having a "partner" pointing in the opposite direction. Figure 4 plots the evolution of the temporal $z$-scores for $N_{\text {core }}$ and $r$. Overall, the two trends show some similarities, being characterized by peaks that correspond to so-called bubbles, i.e., periods of "unsustainable" price growth [36]; interestingly, such periods are characterized by values of the inspected topological quantities which are significant also in a statistical sense, as demonstrated by the values of the corresponding temporal $z$-scores (in fact, $z^{(t)} \geq 2$ in both cases). Moreover, peaks are also revealed in 20142016 , thus signaling some kind of "activity" missed by purely financial indicators (e.g., the RPMA).

\subsection{The Bitcoin Lightning Network}

Let us now move on to results concerning the BLN. In what follows we will focus on the daily-block snapshot representation.

\subsubsection{Basic Statistics}

As observed for the BAN and the BUN, both the number of nodes $N$ and the number of links $L=\sum_{i} \sum_{j(>i)} b_{i j}$ of the BLN increase steadily over time, while the network becomes sparser. Interestingly, however, the evolution of the BLN link density seems to point to the presence of two regimes. As Figure 5 shows, during the first phase (i.e., $N \leq 10^{3}$ ), $L$ increases linearly in $N$ and the link density is well-described by the functional dependence $d \sim N^{-1}$; afterwards, the decrease in link density slows down and seems to indicate that $L$ has started to grow in a super-linear fashion with respect to $N$.

\subsubsection{Analysis of the BLN Mesoscale Structure}

Although blockchain-based systems are designed to eliminate the need for a central authority to check the validity of exchanges between nodes (i.e., transactions in the case of cryptocurrencies) and authorize them, it is shown in Lin et al. [28] that centralization may still be recovered at a purely structural level. More precisely, the authors considered two sets of quantities. First, they computed the Gini coefficient

$$
G_{c}=\frac{\sum_{i=1}^{N} \sum_{j=1}^{N}\left|c_{i}-c_{j}\right|}{2 N \sum_{i=1}^{N} c_{i}}
$$

for four centrality measures, the degree, closeness, betweenness, and eigenvector centrality (respectively denoted by the symbols $c_{i}=k_{i}^{c}, c_{i}^{c}, b_{i}^{c}$, and $e_{i}^{c}$; see also Appendix B and [37]), and plotted it against the number of nodes. As shown in Lin et al. [28], $G_{c}$ increases for three out of the four measures, namely the degree, betweenness, and eigenvector centrality, but not for the closeness centrality, whose trend remains basically flat. Since the Gini coefficient quantifies the (un)evenness of a distribution, this result suggests that the centrality of the nodes is becoming more and more unevenly distributed. A concrete example is provided by the value $G_{k^{c}}$ reaching $\simeq 0.8$ in the last snapshot of our data set; this value is compatible with the picture of a network where $90 \%$ of connections are incident to $10 \%$ of the nodes. In other words, nodes exist that play the role of hubs, i.e., vertices with a large number of connections, which are crossed by a large percentage of paths and are connected to other wellconnected nodes.

Additionally, Lin et al. [28] computed the so-called centralization indices, which encode comparisons between the structure of a given network and that of a reference network, i.e., the "most centralized" structure; see also Appendix B. For the degree, closeness, and betweenness centrality measures, it is the star graph; for the eigenvector index, the star graph does not represent the maximally centralized structure, although it is retained for consistency with the other quantities. The evolution of the centralization 

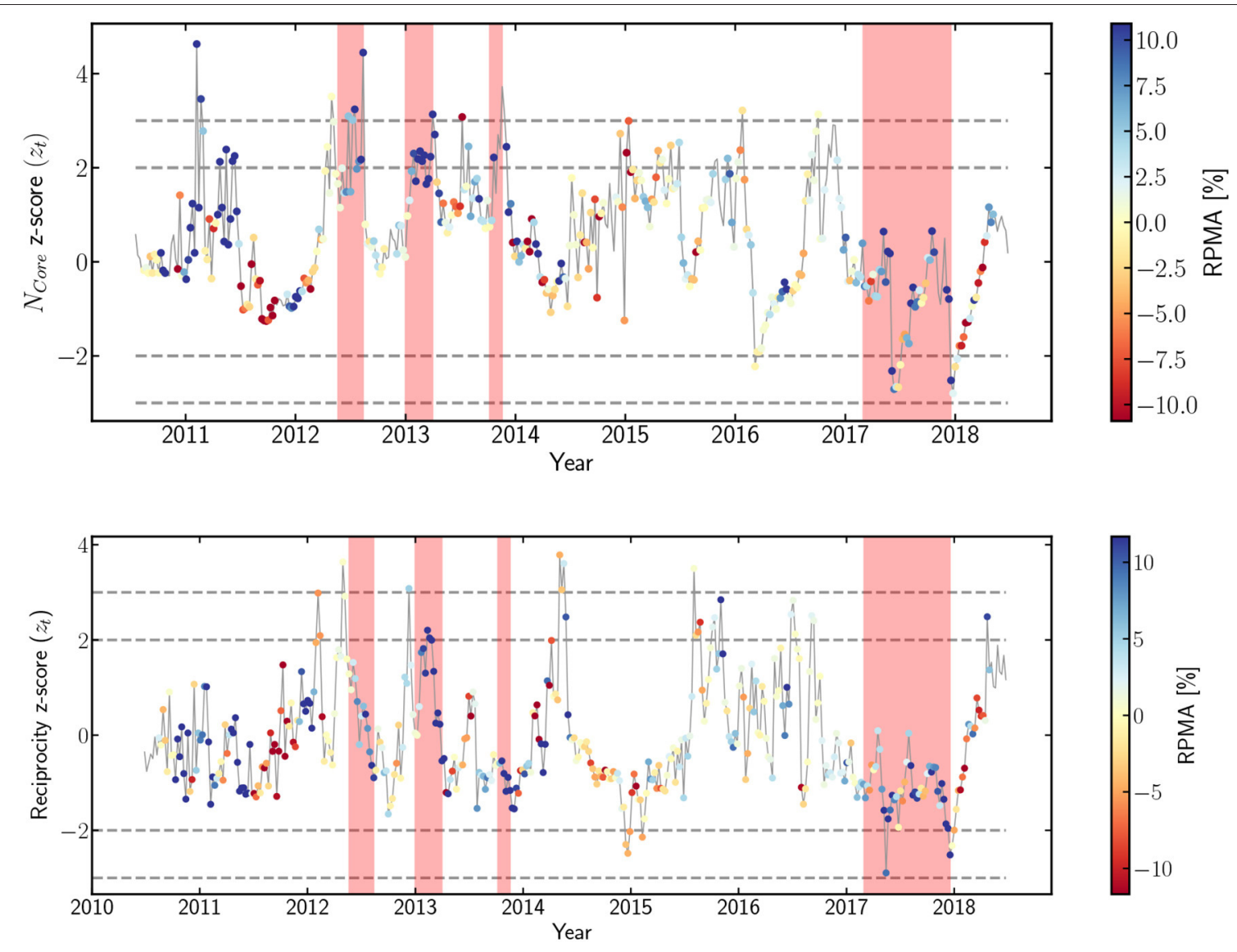

FIGURE 4 | Evolution of the temporal z-score for the number of core nodes (top panel) and the reciprocity (bottom panel) for the BUN weekly representation. Shaded areas indicate so-called bubbles, i.e., periods of price increase according to Wheatley et al. [36]. Additionally, each dot representing an observation is colored according to the value of the ratio between the current price and its moving average (RPMA) indicator. Overall, the two trends show some similarities, with peaks clearly visible in correspondence to so-called bubbles, identified by the shaded areas (see also Wheatley et al. [36]). Interestingly, these quantities are also significant in a statistical sense, as the temporal $z$-scores reach values of $z^{(t)} \geq 2$.

indices indicates that the BLN is evolving not toward a star graph (which is indeed a too-simplistic picture to faithfully describe the BLN topology) but toward a suitable generalization of it, i.e., the core-periphery structure [28] (see also later). Incidentally, the presence of a core-periphery structure is compatible with the aforementioned even distribution of the closeness centrality, since by definition the closeness of a core node does not differ much from the closeness of a periphery node.

In Lin et al. [28] the observations concerning the evolution of the centrality measures and the centralization indices were also benchmarked against the predictions for the same quantities output by the maximum-entropy null model known as the undirected binary configuration model (UBCM; see also Appendix C). To this end, the authors explicitly sampled the ensembles of networks induced by the UBCM [38, 39] and compared the ensemble average of each quantity of interest with the corresponding empirical value. For technical reasons, the authors adopted an iterative, reduced algorithm to solve the system of equations defining the UBCM,

$$
\begin{aligned}
k_{i}(\mathbf{A})= & \sum_{j(\neq i)=1}^{N} \frac{x_{i} x_{j}}{1+x_{i} x_{j}}, \forall i \Longrightarrow \\
x_{k}^{(n)}= & \frac{k(\mathbf{A})}{\sum_{k^{\prime}}\left[f\left(k^{\prime}\right)-\delta_{k k^{\prime}}\right]\left(\frac{x_{k^{\prime}}^{(n-1)}}{1+x_{k}^{(n-1)} x_{k^{\prime}}^{(n-1)}}\right)}, \forall k
\end{aligned}
$$

which enabled them to solve the system of equations within tens of seconds even for configurations with millions of nodes [27]; see also Appendix C. As Figure 6 shows, this comparison reveals that the UBCM tends to overestimate the values of the Gini index for the degree, the closeness, and the betweenness centrality measures and to underestimate the values for the eigenvector centrality. This seems to point to a non-trivial (i.e., not reproducible by just enforcing the degrees) tendency of well-connected nodes to establish 

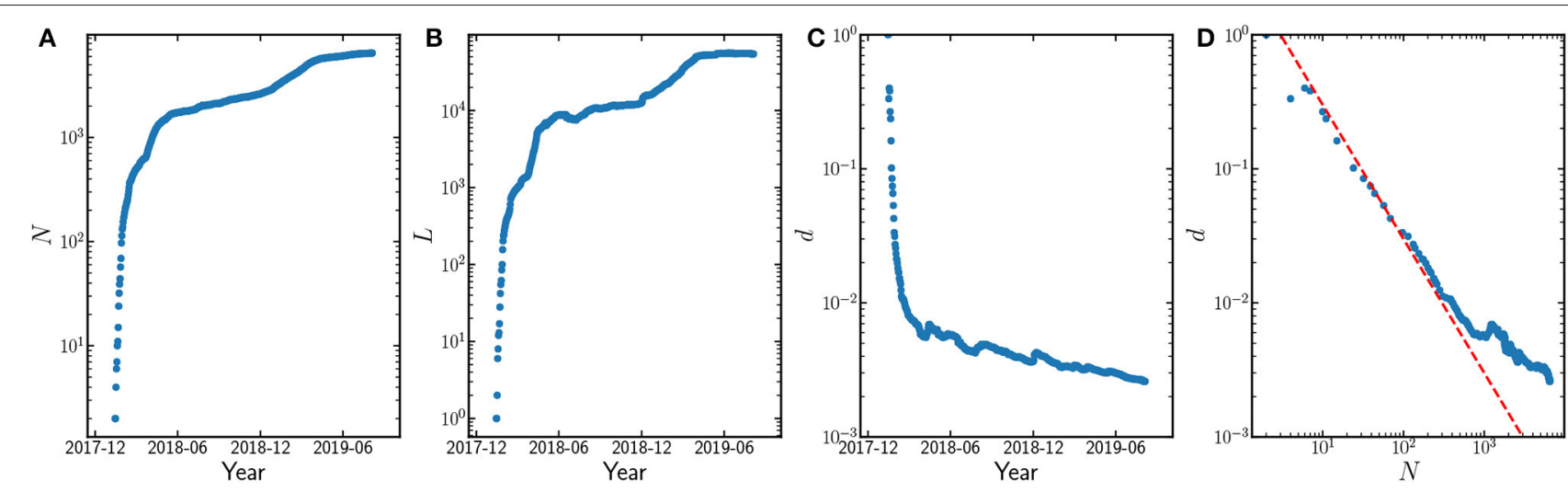

FIGURE 5 | Evolution of the total number of nodes $N$ (A), total number of links $L$ (B) and link density $d=\frac{2 L}{N(N-1)}(\mathbf{C})$ for the BLN daily-block snapshot representation. As for the BAN and the BUN, the position $d \sim N^{-1}$ well describes the link density dependence on $N$ (D), at least for the snapshots for which $N \leq 10^{3}$. See also Lin et al. [28].

connections among themselves-likely with nodes having a smaller degree associated with them. Such a disassortative structure could explain the less-than-expected level of unevenness characterizing the other centrality measures, as the nodes behaving as "leaves" of the hubs would basically have the same values of degree, closeness, and betweenness centrality.

For the analysis of the centralization indices, Figure 6 shows that the UBCM underestimates both the betweenness and the eigenvector centralization indices; in other words, a tendency toward centralization "survives" even after the information encoded in the degrees is properly accounted for, letting the picture of a network characterized by some kind of morethan-expected "star-likeness" emerge. This observation can be better formalized by analyzing the BLN mesoscale structure via optimization of the surprise; as observed for the BUN, a coreperiphery structural organization, whose statistical significance increases over time, indeed emerges [28] (see also Figure 7).

In Bovet et al. [27], the authors also adapted the iterative, reduced algorithm cited above for the resolution of the directed binary configuration model (DBCM; see also Appendix C).

\subsubsection{A Quick Look at the Weighted Structure of the BLN}

A quick look at the weighted structure of the BLN yields two notable observations: both the total amount of exchanged bitcoins and the unevenness of their distribution increase. This trend is confirmed by the evolution the Gini coefficient, whose value reaches 0.9 for the last snapshots of our data set. On average, over the entire period, about $10 \%$ (50\%) of the nodes hold $80 \%(99 \%)$ of the bitcoins at stake in the network [28].

\section{DISCUSSION}

The public availability of the complete history of Bitcoin transactions allows researchers to analyze the structure characterizing different transaction networks, to inspect the inter-dependency between the network dynamics and the Bitcoin price, and to gain insight into the behavior of Bitcoin users. Still, understanding of the mechanisms underlying the joint evolution of these three entities remains far from complete.

This paper provides an overview of the most recent results on the topic. One of the main messages concerns the possibility of retrieving signals of exogenous events by analyzing the blockchain-induced transaction networks; the best example is provided by the failure of Mt. Gox in 2014, an event that strongly affected the structure of both the Bitcoin Address Network and the Bitcoin User Network. From this point of view, out-degrees have been found to represent particularly informative properties: higher moments of the out-degrees distribution (such as the standard deviation, skewness, and kurtosis) diverge as the network size becomes larger than the observed value corresponding to the Mt. Gox failure; moreover, the out-degrees heterogeneity rises during periods of price decline (and vice versa).

This result has been further refined by a Granger causality analysis, revealing that during the years 2010-2012 an increase in the out-degrees standard deviation caused a price decline [26]. This finding, in turn, suggests a sort of behavioral explanation for the price dynamics exhibited in the early stages of Bitcoin: during periods in which the price continuously increased, more traders were attracted to the system; the later-joining ones, likely performing only a few transactions, linked to the network hubs (usually exchange markets), which gained a large number of connections over these weeks, thus causing the price to rise even further.

Interestingly, analysis of the Bitcoin Lightning Network reveals the same trends as observed for the BAN and BUN, namely the emergence of an uneven distribution of the centrality and the wealth of nodes and of a statistically significant coreperiphery structure. These results suggest a tendency of the Bitcoin "Layer 2" network to become less distributed, a process having the undesirable consequence of making this off-chain payment network less resilient to random failures, malicious attacks, etc. The emergence of hubs may be a consequence of 

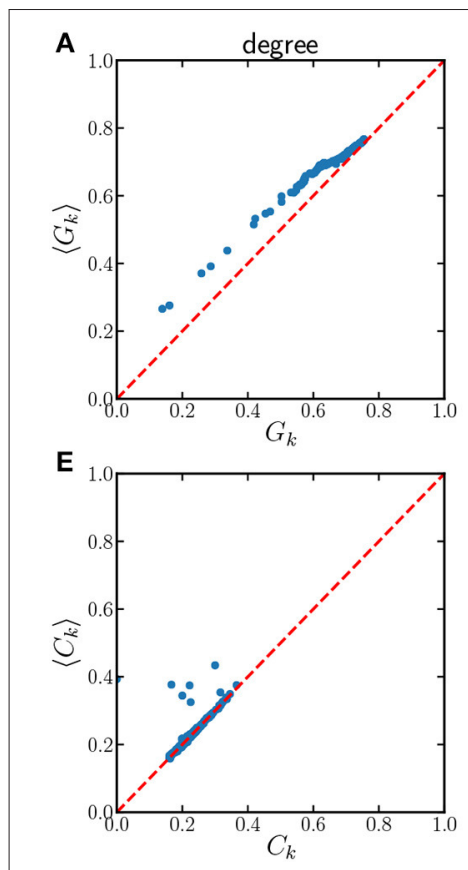
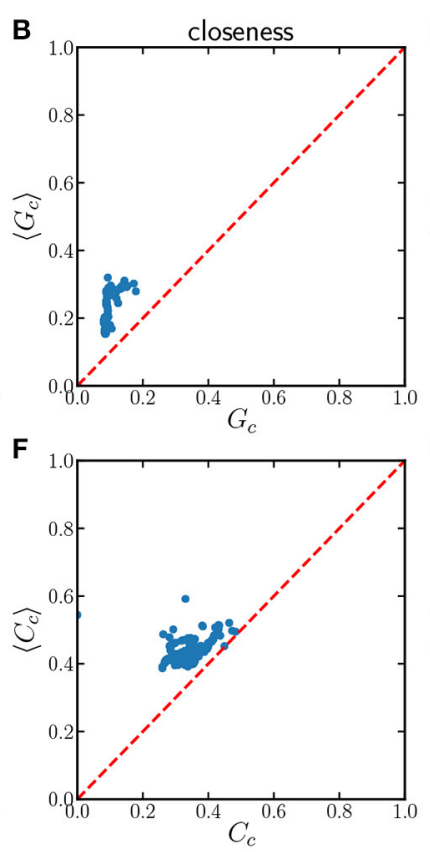
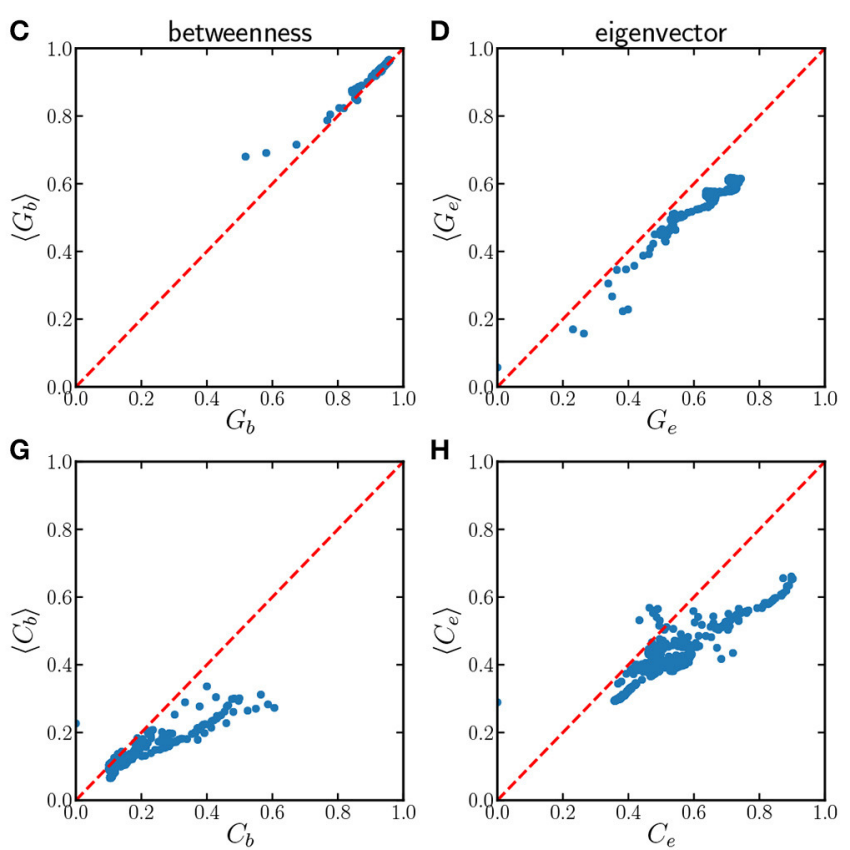

FIGURE 6 | Top panels: comparison between the observed Gini index for the degree (A), closeness (B), betweenness (C) and eigenvector centrality (D) on the X-axis and their expected value, computed under the UBCM (y-axis) for the BLN daily-block snapshot representation. Bottom panels: comparison between the observed degree- (E), closeness- (F), betweenness- (G) and eigenvector- (H) centralisation measures and their expected value computed under the UBCM. Once the information contained into the degree sequence is properly accounted for, a (residual) tendency to centralisation is still visible, letting the picture of a network characterised by some kind of more-than-expected 'star-likeness' emerge. See also [28].

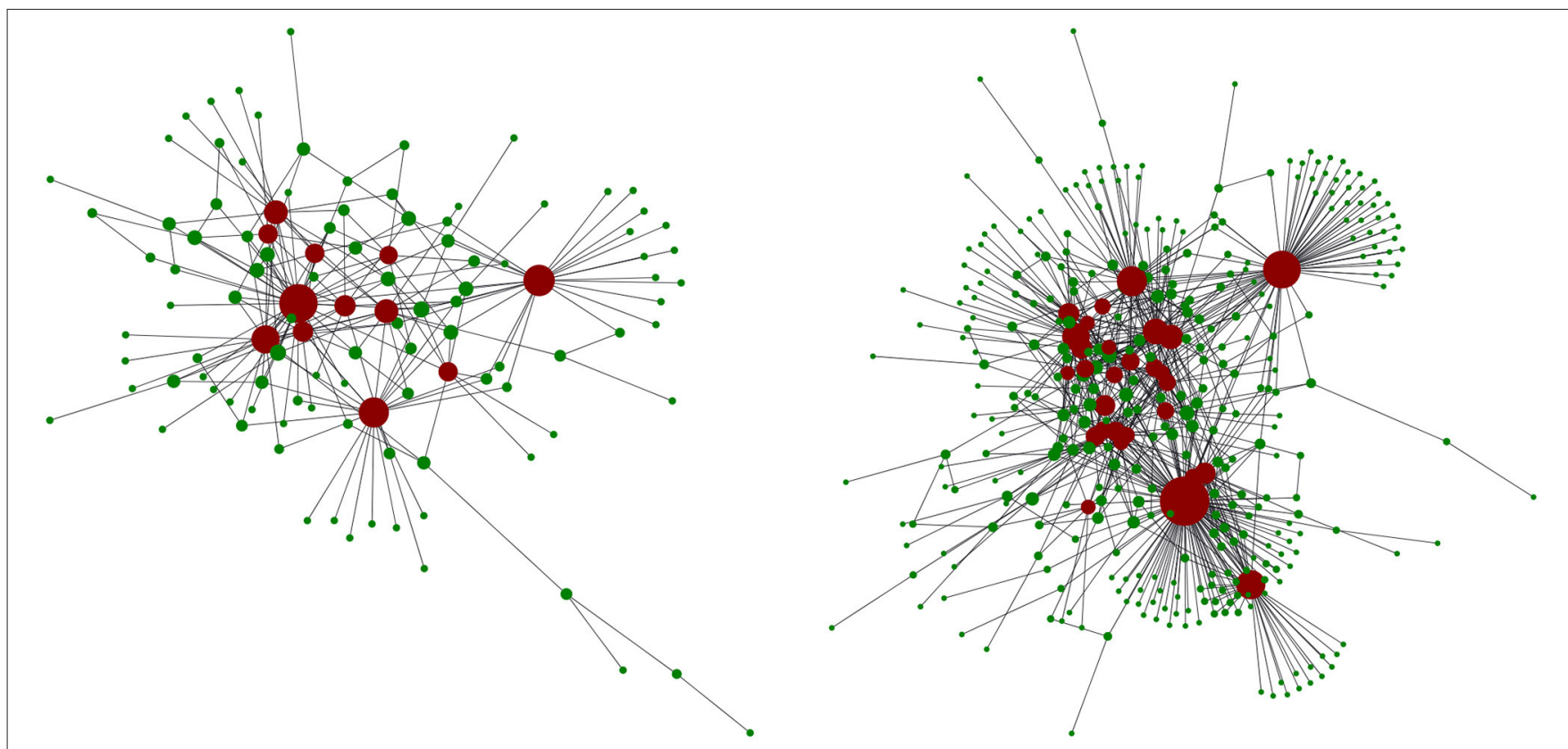

FIGURE 7 | Core-periphery structure of the BLN daily-block representation on day 17 (left panel) and on day 35 (right panel), with core nodes shown in red and periphery nodes in green. Adapted from Lin et al. [28].

the way the BLN is designed: as a route through the network must be found and longer routes are more expensive (fees are charged for the gateway service provided by intermediate nodes), any two BLN users will search for a short(est) path; at the same time, nodes have the incentive to become as central as possible, in order to maximize the transaction fees they can earn. Hubs 
may thus have emerged as a consequence of the collective action of users exhibiting one of the two aforementioned behaviorsnot surprisingly, since the very beginning of the BLN's history. Regarding the interconnectedness of hubs, previous results have shown that mechanisms aimed at maximizing the centrality of agents give rise to a core-periphery structure (regardless of the notion of centrality the agents attempt to maximize) [40, 41]. As a final remark, we also note that the presence of "centrality hubs" seems to be the source of another peculiarity of the BLN structure, i.e., its small-worldness (a feature already revealed by previous studies [42]).

The results reviewed in this article ultimately-and consistently-point to a tendency toward centralization, which has been observed in the Bitcoin network structure at different levels $[28,43]$ and is evidence that deserves to be investigated in greater detail. A natural extension of the present work is to analyze the weighted counterparts of the three constructs considered here. Of particular interest would be analysis of the weighted centrality measures and centralization indices considered in Lin et al. [28], the outcome of which would help clarify to what extent the observation that binary and weighted quantities are usually correlated in financial systems holds true for cryptocurrencies as well. Other promising avenues of research concern the analysis of different cryptocurrencies

\section{REFERENCES}

1. Antonopoulos M. Mastering Bitcoin: Programming the Open Blockchain. Sebastopol, CA: O'Reilly Media, Inc. (2017).

2. Nakamoto S. Bitcoin: A Peer-to-Peer Electronic Cash System. (2008).

3. Halaburda H, Sarvary M. Beyond Bitcoin: The Economics of Digital Currencies. Basingstoke: Palgrave Macmillan (2016).

4. Glaser F. In: Proceedings of the Hawaii International Conference on System Sciences 2017 (HICSS-50). (2017)

5. Decker C, Wattenhofer R. Decision analytics, mobile services, and service science In: IEEE P2P 2013 Proceedings. Waikoloa Village, HI: IEEE (2013). p. $1-10$.

6. Poon J, Dryja T. The Bitcoin Lightning Network: Scalable Off-Chain Instant Payments. Trento. (2006).

7. Hencic A, Gourieroux C. Econometrics of Risk. Cham: Springer (2014).

8. Chu J, Nadarajah S, Chan S. Statistical analysis of the exchange rate of bitcoin. PLoS ONE. (2015) 10:e0133678. doi: 10.1371/journal.pone.0133678

9. Chan S, Chu J, Nadarajah S, Osterrieder J. A statistical analysis of cryptocurrencies. J Risk Financ Manag. (2017) 10:12. doi: $10.3390 /$ jrfm 10020012

10. Sapuric S, Kokkinaki A. DC workshop. In: Business Information Systems Workshops, Lecture Notes in Business Information Processing. Larnaca: Springer (2014).

11. Briere M, Oosterlinck K, Szafarz A. Virtual currency, tangible return: portfolio diversification with bitcoin. J Asset Manag. (2015) 16:365-73. doi: $10.1057 /$ jam.2015.5

12. Kristoufek A. What are the main drivers of the bitcoin price? Evidence from wavelet coherence analysis. PLoS ONE. (2015) 10:e0123923. doi: 10.1371/journal.pone.0123923

13. Yiying W. Cryptocurrency price analysis with artificial intelligence. In: Proceedings of the 5th International Conference on Information Management Cryptocurrency Price Analysis With Artificial Intelligence. Cambridge: IEEE (2019). doi: 10.1109/INFOMAN.2019.8714700

14. Garcia D, Tessone CJ, Mavrodiev P, Perony N. The digital traces of bubbles: feedback cycles between socio-economic signals in the and other blockchain-based systems, to understand whether the mechanisms shaping the Bitcoin structure are also at work elsewhere.

\section{AUTHOR CONTRIBUTIONS}

CJT and TS designed the review structure. NV performed the additional analyses. NV and TS wrote the manuscript. All authors reviewed and approved the manuscript.

\section{ACKNOWLEDGMENTS}

CJT acknowledges financial support from the University of Zurich through the University Research Priority Program on Social Networks. TS acknowledges support from the EU project SoBigData-PlusPlus (grant no. 871042). The authors thank A. Bovet, C. Campajola, F. Mottes, V. Restocchi, and J.-H. Lin for useful discussions.

\section{SUPPLEMENTARY MATERIAL}

The Supplementary Material for this article can be found online at: https://www.frontiersin.org/articles/10.3389/fphy. 2020.00286/full\#supplementary-material

Bitcoin economy. J R Soc Interface. (2014) 11:99. doi: 10.1098/rsif.2014. 0623

15. El Bahrawy A, Alessandretti L, Baronchelli A. Wikipedia and digital currencies: interplay between collective attention and market performance. Front Blockchain. (2019) 2:1-13. doi: 10.2139/ssrn.3346632

16. Androulaki E, Karame GO, Roeschlin M, Scherer T, Capkun S. Electronic payment (Bitcoin). In: International Conference on Financial Cryptography and Data Security. Okinawa: Springer (2013). p. 34-51. doi: 10.1007/978-3-642-39884-1_4

17. Harrigan M, Fretter C. Cryptocurrencies and blockchain based systems. In: Ubiquitous Intelligence \& Computing, Advanced and Trusted Computing, Scalable Computing and Communications, Cloud and Big Data Computing, Internet of People, and Smart World Congress (UIC/ATC/ScalCom/CBDCom/IoP/SmartWorld), 2016 Intl IEEE Conferences. Toulouse: IEEE (2016). p. 368-73.

18. Meiklejohn S, Pomarole M, Jordan G, Levchenko K, McCoy D, Voelker GM, et al. In: Proceedings of the 2013 Conference on Internet Measurement Conference. ACM (2013). p. 127-40.

19. Ober M, Katzenbeisser S, Hamacher K. Structure and anonymity of the Bitcoin transaction graph. Fut Internet. (2013) 5:237-50. doi: 10.3390/fi5020237

20. Reid F, Harrigan M. Security and Privacy in Social Networks. Springer (2013).

21. Kondor D, Csabai I, Vattay G. Do the rich get richer? An empirical analysis of the Bitcoin transaction network. PLoS ONE. (2014) 9:e0086197. doi: 10.1371/journal.pone.0086197

22. Javarone MA, Wright CS. From Bitcoin to Bitcoin cash: a network analysis. arXiv. (2018) 1804.02350v2. doi: 10.1145/3211933.3211947

23. Parino F, Beiró MG, Gauvin L. Analysis of the Bitcoin blockchain: socioeconomic factors behind the adoption. Eur Phys J Data Sci. (2018) 7:38. doi: 10.1140/epjds/s13688-018-0170-8

24. Motamed AP, Bahrak B. Quantitative analysis of cryptocurrencies transaction graph. Appl Netw Sci. (2019) 4:131. doi: 10.1007/s41109-0190249-6

25. Liang J, Li L, Zeng D. Evolutionary dynamics of cryptocurrency transaction networks: an empirical study. PLoS ONE. (2018) 13:e0202202. doi: 10.1371/journal.pone.0202202 
26. Bovet A, Campajola C, Mottes F, Restocchi V, Vallarano N, Squartini T, et al. The evolving liaisons between the transaction networks of Bitcoin and its price dynamics. arXiv. (2019) 1907.03577.

27. Bovet A, Campajola C, Lazo JF, Mottes F, Pozzana I, Restocchi V, et al. Network-based indicators of Bitcoin bubbles. arXiv. (2020) 1805.04460.

28. Lin JH, Primicerio K, Squartini T, Decker C, Tessone CJ. Lightning Network: a second path towards centralization of the Bitcoin economy. arXiv. (2020) 2002.02819. doi: 10.1088/1367-2630/aba062

29. Tasca P, Hayes A, Liu S. The evolution of the Bitcoin economy: extracting and analyzing the network of payment relationships. J Risk Finance. (2018) 19:94-126. doi: 10.1108/JRF-03-2017-0059

30. Ron D, Shamir A. Electronic payment (Bitcoin). In: International Conference on Financial Cryptography and Data Security. Okinawa: Springer (2013). p. 6-24. doi: 10.1007/978-3-642-39884-1_2

31. Baumann A, Fabian B, Lischke M. WEBIST. Barcelona: SciTePresss (2014).

32. Bauke H. Parameter estimation for power-law distributions by maximum likelihood methods. Eur Phys J B. (2007) 58:167-73. doi: 10.1140/epjb/e2007-00219-y

33. Bianconi G, Barabasi S. Bose-einstein condensation in complex networks. Phys Rev Lett. (2001) 86:5632. doi: 10.1103/PhysRevLett.86.5632

34. Granger W. Investigating causal relations by econometric models and crossspectral methods. Econometrica. (1969) 37:424-38. doi: 10.2307/1912791

35. de Jeude JvL, Caldarelli G, Squartini T. Detecting core-periphery structures by surprise. Europhys Lett. (2019) 125:68001. doi: 10.1209/0295-5075/125/68001

36. Wheatley S, Sornette D, Huber T, Reppen M, Gantner RN. Are Bitcoin Bubbles Predictable? Combining a Generalized Metcalfe's Law and the LPPLS Model. Zurich: Swiss Finance Institute Research Paper (2018).

37. Wang J, Li C, Xia C. Improved centrality indicators to characterize the nodal spreading capability in complex networks. Appl Math Comput. (2018) 334:388-400. doi: 10.1016/j.amc.2018.04.028
38. Park J, Newman ME. Statistical mechanics of networks. Phys Rev E. (2004) 70:066117. doi: 10.1103/PhysRevE.70.066117

39. Squartini T, Garlaschelli D. Analytical maximum-likelihood method to detect patterns in real networks. New J Phys. (2011) 13:083001. doi: 10.1088/1367-2630/13/8/083001

40. König MD, Tessone CJ, Zenou Y. Nestedness in networks: a theoretical model and some applications. CEPR Discuss Pap. (2014) 9:695-752. doi: 10.3982/TE1348

41. König MD, Tessone CJ, Zenou Y. From assortative to dissortative networks: the role of capacity constraints. Adv Complex Syst. (2010) 13:483. doi: 10.1142/S0219525910002700

42. Rohrer E, Malliaris J, Tschorsch F. Discharged payment channels: quantifying the lightning network's resilience to topology-based attacks. arXiv. (2019) 1904.10253. doi: 10.1109/EuroSPW.2019. 00045

43. Gervais A, Karame G, Capkun S, Capkun V. Is Bitcoin a decentralized currency? IEEE Sec Priv. (2014) 12:54-60. doi: 10.1109/MSP.20 14.49

Conflict of Interest: The authors declare that the research was conducted in the absence of any commercial or financial relationships that could be construed as a potential conflict of interest.

Copyright (c) 2020 Vallarano, Tessone and Squartini. This is an open-access article distributed under the terms of the Creative Commons Attribution License (CC BY). The use, distribution or reproduction in other forums is permitted, provided the original author(s) and the copyright owner(s) are credited and that the original publication in this journal is cited, in accordance with accepted academic practice. No use, distribution or reproduction is permitted which does not comply with these terms. 